\title{
SURVEILLANCE OF CATHETER-RELATED BLOODSTREAM INFECTIONS AMONG ADULT PATIENTS IN ICU
}

\author{
Poulami P1, Yashavanth $R^{2}$, Narendra $N^{3}$
}

${ }^{1}$ Resident Medical Officer, Narayana Super Speciality Hospital, Howrah.

${ }^{2}$ Associate Professor, Department of Microbiology, A. J. Institute of Medical Sciences \& Research Centre, Mangaluru.

${ }^{3}$ Professor, Department of Microbiology, A. J. Institute of Medical Sciences \& Research Centre, Mangaluru.

\section{ABSTRACT}

\section{BACKGROUND}

Catheter-related bloodstream infections (CRBSI) are an important contributor for the increasing morbidity and mortality in patients on central venous catheter (CVC). The incidence of CRBSI increases with the duration of catheterisation. Hence, it requires a rapid diagnosis of CRBSI and initiation of an appropriate antibiotic therapy, if not it will lead to emergence of drug resistance and poor prognosis in these patients.

Aims and Objectives - The study was undertaken to confirm the CRBSI and to identify aerobic bacterial pathogens and evaluating their antimicrobial susceptibility pattern.

\section{MATERIALS AND METHODS}

The study was conducted on 150 admitted patients with indwelling Central Venous Catheter (CVC). Two sets of blood samples were collected, one each from CVC line and another from peripheral vein in BACTEC bottle and incubated in BD BACTEC 9050 system. CRBSI was diagnosed if same organism was isolated from both samples. Antibiotic susceptibility testing was done for those isolates.

\section{RESULTS}

CRBSI rate was found to be 2.81 per 1000 catheter days. The incidence of aerobic Gram-negative bacteria was $66.7 \%$ (Klebsiella pneumoniae and Serratia marcescens) and Gram-positive cocci were 33.3\% (Staphylococcus aureus).

\section{CONCLUSION}

According to National Nosocomial Infections Surveillance (NNIS) System report, range should be within 2 to 11.3 per 1000 catheter days. In our study, it is 2.81 per 1000 catheter days, which falls within the range as per NNIS. There is a need for a prospective study in India so that it can help in formulating infection control policies to prevent catheter-related blood stream infections.

\section{KEYWORDS}

Central Venous Catheters, Blood Stream Infections, Antibiotic Susceptibility Testing.

HOW TO CITE THIS ARTICLE: Poulami P, Yashavanth R, Narendra N. Surveillance of Catheter-related bloodstream infections among adult patients in ICU. J. Evolution Med. Dent. Sci. 2017;6(2):118-123, DOI: 10.14260/Jemds/2017/30

\section{BACKGROUND \\ Catheter-related bloodstream infections (CRBSIs) most of which are associated with central venous catheters (CVCs) accounts for $11 \%$ of all Healthcare Associated Infections (HAIs). Centres for Disease Control and Prevention (CDC) were informed in response to the growing awareness that HAIs are urgent public health and patient safety issue.[1] Blood stream infections (BSIs) which represent the failure of immune system to contain infection at a focal site and consequent disseminated disease are a major cause of morbidity and mortality in the world. Currently, such infections are the $13^{\text {th }}$ leading cause of death and over the past 2 decades the death rate from septicaemia has risen by $8 \% .{ }^{[2]}$}

Financial or Other, Competing Interest: None.

Submission 30-11-2016, Peer Review 23-12-2016,

Acceptance 30-12-2016, Published 05-01-2017.

Corresponding Author:

Dr. Yashavanth $R$

Associate Professor,

Department of Microbiology,

A.J. Institute of Medical Sciences \& Research Centre,

Kuntikana, Bejai, Mangaluru, Karnataka.

E-mail: dryashwanthrai@gmail.com

DOI: $10.14260 /$ jemds $/ 2017 / 30$

\section{(c) $(1) \ominus$}

Nosocomial blood stream infections in patients in the intensive care unit (ICU) are associated and attributable to the mortality rate of $35 \% .^{[3]}$ Patients on central venous catheters pose a greater risk of device related infection than any other type of medical device and also main source of bacteraemia and septicaemia in hospitalised patients. ${ }^{[4]}$ The continued presence of foreign body and placement of the device through the skin and into the blood vessels bypasses one of the body's best barriers to infections and creates the potential for microorganisms to enter the bloodstream and predispose further to infection, which can result from colonisation of the catheters by the skin flora or less commonly by haematogenous seeding due to hub contamination.[5] Early detection and adequate treatment of pathogen causing central venous catheter infections should be done within 24 hours of clinically suspicious symptoms for favourable outcome as these infections develop when catheters are left in place for a period more than 48 hours, because of excessive manipulation of catheter hub and forgetting to disinfect the intravenous access port prior to infusion leading to contamination of the internal lumen. [6,7]

Gram-positive organisms accounted for $64 \%$ of cases, Gram-negative organism which accounted for $28 \%$ and $8 \%$ were caused by fungi.[2] The most common organisms were coagulase negative Staphylococcus (CONS), Staphylococcus 


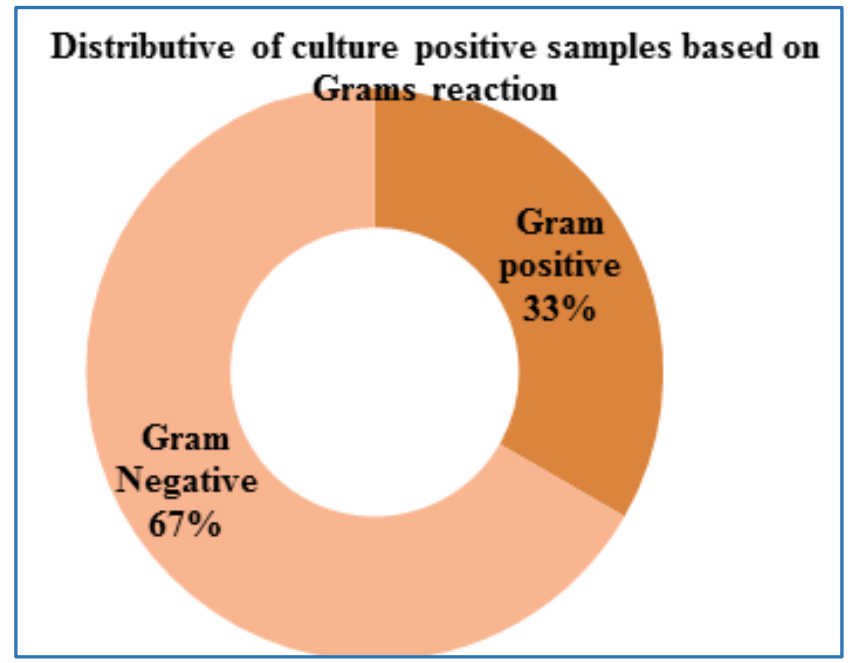

Figure 2. Distribution of Culture-positive samples based on Gram Reaction

Out of 3 positive cases in our study, male predominance was more accounting for $66.7 \%$ whereas female accounted for only $33.3 \%$. (Table 2)

\begin{tabular}{|c|c|c|c|}
\hline \multirow{2}{*}{ Gender } & Growth of Organisms & \multirow{2}{*}{ Total } \\
\cline { 2 - 3 } & Growth & No Growth & \\
\hline Female & $1(33.3 \%)$ & $50(34 \%)$ & $51(34 \%)$ \\
\hline Male & $2(66.7 \%)$ & $97(65 \%)$ & $99(66 \%)$ \\
\hline Total & $\mathbf{3 ( 1 0 0 \% )}$ & $147(100 \%)$ & $150(100 \%)$ \\
\hline \multicolumn{3}{|c|}{ Table 2. Gender-wise Distribution of Blood Culture-positive } \\
Cases \\
\hline
\end{tabular}

$\mathrm{X}^{2}=0.0603, \mathrm{p}=0.90, \mathrm{~ns}$, Chi-square test.

Out of 150 blood samples, 90.7\% was taken from MICU, 8.7\% from SICU and 0.6\% from CCU. (Table 3 \& Fig 3).

\begin{tabular}{|c|c|c|}
\hline & Frequency & Percentage \\
\hline MICU & 136 & 90.7 \\
\hline SICU & 13 & 8.7 \\
\hline CCU & 1 & 0.6 \\
\hline Total & $\mathbf{1 5 0}$ & $\mathbf{1 0 0 . 0}$ \\
\hline \multicolumn{3}{|c|}{ Table 3. Ward wise Distribution } \\
\hline
\end{tabular}

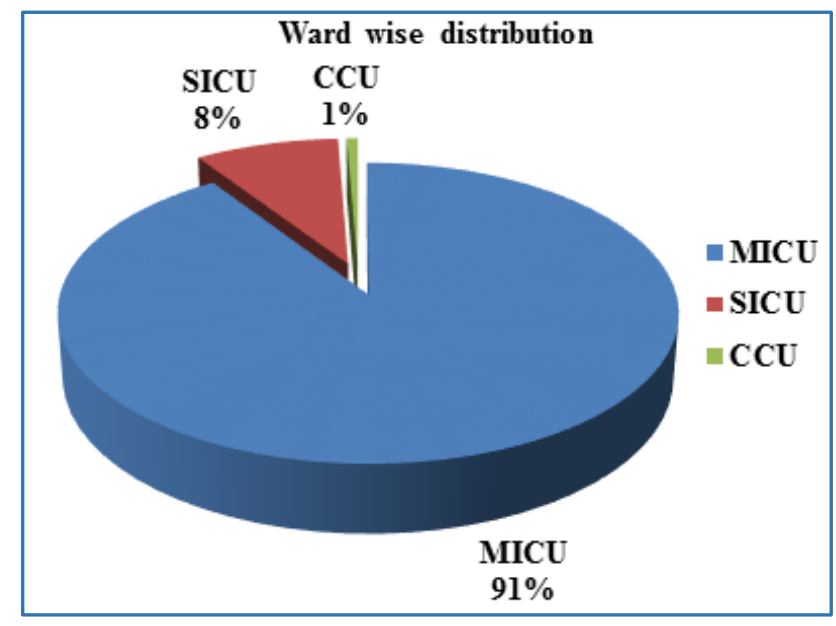

Figure 3. Ward wise Distribution

Among all 150 catheter samples collected for study, 28 (18.7\%) were from age group 40-50 years, in 20-30 years and
50-60 years 27 (18\%) each, $26(17.3 \%)$ from age group 6070 years. Out of 3 culture-positive cases, 1 (33.33\%) was from age group 20-30 years, 1 (33.33\%) was from age group $30-40$ years and $1(33.33 \%)$ was from age group $60-70$ years. (Table 4 \& Fig 4).

\begin{tabular}{|c|c|c|c|}
\hline \multirow{2}{*}{ Age in Years } & Growth of Organism & \multirow{2}{*}{ Total } \\
\cline { 2 - 3 } & Growth & No Growth & \\
\hline$<20$ & 0 & $6(4.1 \%)$ & $6(4 \%)$ \\
\hline $20-30$ & $1(33.33 \%)$ & $26(17.7 \%)$ & $27(18 \%)$ \\
\hline $30-40$ & $1(33.33 \%)$ & $19(12.9 \%)$ & $20(13.3)$ \\
\hline $40-50$ & $0(0 \%)$ & $28(19 \%)$ & $28(18.7 \%)$ \\
\hline $50-60$ & $0(0 \%)$ & $27(18.4 \%)$ & $27(18 \%)$ \\
\hline $60-70$ & $1(33.33 \%)$ & $25(17 \%)$ & $26(17.3 \%)$ \\
\hline$>70$ & $0(0 \%)$ & $16(10.9 \%)$ & $16(10.7 \%)$ \\
\hline Total & $3(100 \%)$ & $147(100 \%)$ & $150(100 \%)$ \\
\hline \multicolumn{3}{|c|}{ Table 4. Age wise Distribution } \\
\hline
\end{tabular}

$\mathrm{X}^{2}=3.34, \mathrm{p}=0.765$, ns, Chi-square test.

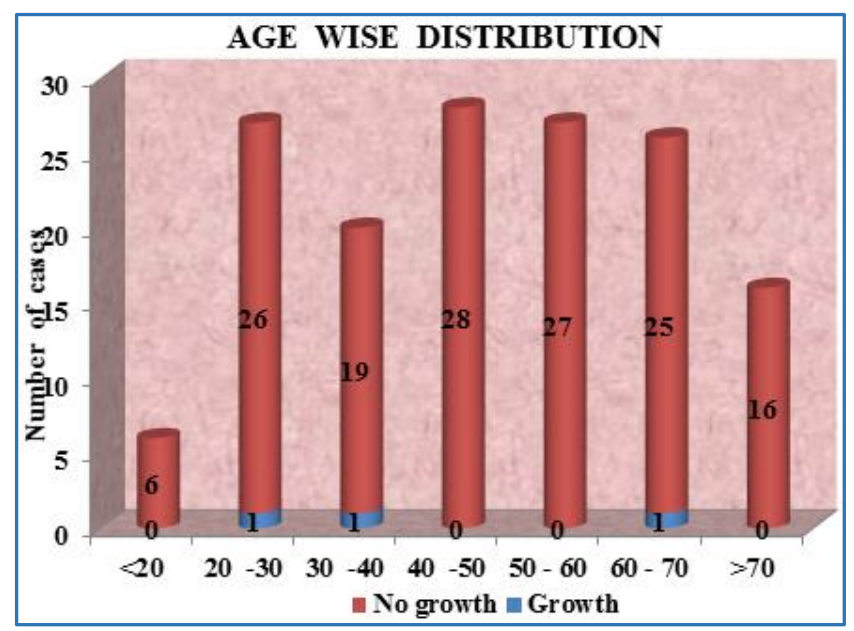

Figure 4. Age wise Distribution

Among all samples, 66 (44\%) were collected from internal jugular vein, 65 (43.3\%) were from femoral vein and $19(12.7 \%)$ were from subclavian vein. Out of these, $1.5 \%$ and $3 \%$ were culture positive from internal jugular vein and femoral vein respectively. (Table 5).

\begin{tabular}{|c|c|c|c|}
\hline \multirow{2}{*}{ Site of Insertion } & \multicolumn{2}{|c|}{ Growth of Organisms } & \multirow{2}{*}{$\begin{array}{c}\text { Total } \\
\text { (n=150) }\end{array}$} \\
\cline { 2 - 3 } & Growth & No. Growth & $66(44 \%)$ \\
\hline IJV & $1(1.5 \%)$ & $65(98.5 \%)$ & $65(43.3 \%)$ \\
\hline Femoral vein & $2(3 \%)$ & $63(97 \%)$ & $65(12.7 \%)$ \\
\hline Subclavian vein & $0(0 \%)$ & $19(100 \%)$ & $19(12)$. \\
\hline \multicolumn{3}{|c|}{ Table 5. Place of Insertion } \\
\hline
\end{tabular}

Out of total 3 positive cases, one Klebsiella pneumoniae, one Serratia marcescens and one S. aureus were isolated. (Table 6)

\begin{tabular}{|c|c|c|}
\hline & No. of Isolates & Percentage \\
\hline Klebsiella pneumoniae & 1 & 0.7 \\
\hline Serratia marcescens & 1 & 0.7 \\
\hline S. aureus & 1 & 0.7 \\
\hline NG & 147 & 98.0 \\
\hline Total & 150 & 100.0 \\
\hline \multicolumn{3}{|c|}{ Table 6. Pattern of Organism Isolated } \\
\hline
\end{tabular}

According to National Nosocomial Infection Surveillance System (NNIS) report, CRBSI rate should be within 2 to 11.3 
per 1000 catheter days. From this graph, we can say that CRBSI rate of our hospital is within limits. (Fig. 5).

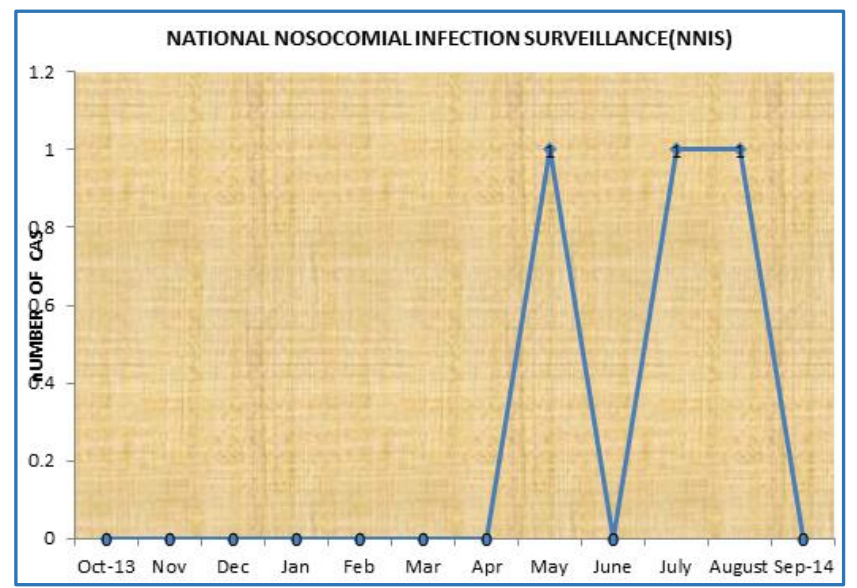

Figure 5. CRBIs rate as per NNIS

\begin{tabular}{|c|c|c|c|c|c|c|c|c|c|c|}
\hline GPC & CX & P & Cot & Cd & E & Cip & Gen & Va & Lz & Te \\
\hline $\begin{array}{c}\text { S. } \\
\text { aureus }\end{array}$ & R & R & S & S & S & S & S & S & S & R \\
\hline
\end{tabular}

$\mathrm{S}=$ Sensitive, $\mathrm{R}=$ Resistant

Staphylococcus aureus showed sensitivity to cotrimoxazole, clindamycin, erythromycin, ciprofloxacin, gentamicin, vancomycin, linezolid and showed resistance to cefoxitin, penicillin, and tetracycline, which was MethicillinResistant Staphylococcus aureus (MRSA). (Table: 7).

\begin{tabular}{|c|c|c|c|c|c|c|c|c|c|c|c|c|c|c|}
\hline GNB & Amp & Gen & Amc & Pi & Pit & Cpm & Ctr & Ctx & Cfs & Caz & Cip & Le & Imp & Mrp \\
\hline Klebsiella pneumoniae & $\mathrm{R}$ & $\mathrm{R}$ & $\mathrm{R}$ & $\mathrm{R}$ & $\mathrm{R}$ & $\mathrm{R}$ & $\mathrm{R}$ & $\mathrm{R}$ & $\mathrm{R}$ & $\mathrm{R}$ & $\mathrm{R}$ & $\mathrm{R}$ & $\mathrm{S}$ & $\mathrm{R}$ \\
\hline Serratia marcescens & $\mathrm{R}$ & $\mathrm{S}$ & $\mathrm{R}$ & $\mathrm{S}$ & $\mathrm{S}$ & $\mathrm{S}$ & $\mathrm{S}$ & $\mathrm{S}$ & $\mathrm{S}$ & $\mathrm{S}$ & $\mathrm{S}$ & $\mathrm{S}$ & $\mathrm{S}$ & $\mathrm{S}$ \\
\hline
\end{tabular}

S=Sensitive, $\mathrm{R}=$ Resistant

Klebsiella pneumoniae was sensitive only to imipenem and resistant to ampicillin, gentamicin, amoxyclav, piperacillin, piperacillin-tazobactam, cefepime, ceftriaxone, cefotaxime, cefoperazone-sulbactam, ceftazidime, ciprofloxacin, levofloxacin and meropenem.

Serratia marcescens was sensitive to ampicillin, gentamicin, piperacillin, piperacillin-tazobactam, cefepime, ceftriaxone, cefotaxime, ceftazidime, cefoperazone-sulbactam, ciprofloxacin, levofloxacin, imipenem, meropenem and resistant to ampicillin, and amoxyclav (Table: 8).

\section{DISCUSSION}

Catheter-related bloodstream infection (CRBSI) is one of the most frequent, lethal and costly complications of central venous catheterisation and also most common cause of nosocomial bacteraemia compounded by the emerging antibiotic resistances that were effective earlier.

In this study, total of 150 suspected cases in 1064 catheter days were observed and CRBI rates were 2.81 per 1000 catheter days. This is comparable to the National Nosocomial Infection Surveillance (NNSI) System report (2 to 11.3 per 1000 catheter days).[13] Similarly, CRBSI rate in the study of S. Singh et al[14] showed 0.48 per 1000 catheter days, Deepti et al[15] showed 8.2 per 1000 catheter days, Ramanathan Parameswaran et al[16] was 8.75 per 1000 catheter days, Daniela Bicudo[17] showed 10.22 per 1000 catheter days, M. Kaur[18] showed 14.59 per 1000 catheter days, Harsha V Patil[19] showed 47.31 per 1000 catheter days. Total 150 catheter samples included in this study. Out of those 3 samples were positive for bacterial growth, so the culture-positive rate is $2 \%$. Studies conducted by S. Singh et al[14] showed $0.8 \%$, Adolf W. Karchma[8] showed $15.68 \%$, Ann 0 Connor et al[20] showed $11.2 \%$ and M. Kaur et al[18] showed $21.73 \%$ of culture positivity. Out of these studies, last three studies reported high culture-positive rates. Culturepositive rate may have a wide range, because it depends on various factors like type of catheters, site of insertion and catheter care. The CRBSI rate varies from hospital to hospitals. It depends on socioeconomic status of patients who were admitted in the hospital, various predisposing factors like diabetics, hypertension, cancers, lymphomas, steroid therapy, chemotherapy, aseptic measures followed by hospitals, diagnostic criteria of CRBSI and protocol of antibiotic used. In our hospital, all these parameters were properly followed by doctors, nurses and staff, so CRBSI rate is low.

In our study, total three culture-positive cases were reported, 1 (33.33\%) from age group 20-30 years, 1 $(33.33 \%)$ from age group $30-40$ years and $1(33.33 \%)$ from age group 60-70 years. Other studies conducted by $\mathrm{H}$. V Patil,[19] Deepti et al,[15] showed high CRBSI rate among 50-60 years of age group whereas study done by Manjula et al[21] showed high culture positivity rate between 19-30 years of age group.

According to this study, male predominance was more which accounted for $66.7 \%$ compared to female $33.3 \%$. Other studies also showed male predominance like the study done by H.V Patil[19] which showed $59 \%$ males and $41 \%$ females were affected, the study done by Deepti et al[15] showed 69\% males and 31\% females affected, the study done by Daniela Bicudo[17] showed 58.85 males and $41.2 \%$ females affected and the study by Ramanathan Parameswaran et al[16] showed $72 \%$ males and $28 \%$ females were affected. CRBSI rates were high in all these studies because of associated or predisposing factors which were seen more in males like smoking, alcohol intake and comorbid illness like HTN, DM, ketoacidosis bronchial asthma, COPD, renal failure, patient on immune suppressive therapy, chemotherapy, etc.

In our study, $90.7 \%$ of the samples were from medical intensive care unit (MICU), 8.7\% from surgical intensive care unit (SICU) and $0.6 \%$ from cardiac care unit (CCU). In the study by S. Singh et al,[14] $12 \%$ of the blood samples were collected from patients admitted in MICU and 88\% from SICU. 
In our study, 66 (44\%) blood samples were collected from internal jugular vein (IJV), 65 (43.3\%) were collected from femoral vein and $19(12.7 \%)$ were collected from subclavian vein. The study conducted by Harsha V Patil[19] showed that $22.22 \%, 38.89 \%$ and $38.89 \%$ CVC were placed into internal jugular vein, femoral vein and subclavian vein respectively. The study conducted by Ramanathan Parameswaran[16] showed that $22.2 \%, 33.3 \%$ and $23 \%$ CVC were placed into internal jugular vein, femoral vein and subclavian vein respectively.

In our study, all 150 patients were on empirical antibiotic therapy. Out of those, three (2\%) patients were positive for CRBSI. That means empirical antibiotics showed high protection from CRBSI. Similarly, S. Singh et al[14] also showed that systemic empirical antibiotic therapy provided high protection from CRBSI.

From the three culture-positive cases, two were Gramnegative bacilli and one Gram-positive cocci; Gram-negative bacilli were Klebsiella pneumoniae (33.33\%), Serratia marcescens (33.33\%) and Gram-positive cocci was Staphylococcus aureus (33.33\%). Similar study done by Deepti et al[15] showed Methicillin-Resistant Staphylococcus aureus(45\%), Klebsiella pneumoniae (11\%), followed by MSSA, Enterococci and Acinetobacter spp.

In this study, Klebsiella pneumoniae was sensitive only to imipenem. Serratia marcescens was sensitive to ampicillin, gentamicin, piperacillin, piperacillin-tazobactam, cefepime, ceftriaxone, cefotaxime, ceftazidime, cefoperazone-sulbactam, ciprofloxacin, levofloxacin, imipenem, meropenem and resistant to ampicillin and amoxyclav. Study done by H.V Patil[19] also showed that Klebsiella pneumoniae was resistant to all antibiotics except fluoroquinolone and carbapenem.

In this study, Staphylococcus aureus was sensitive to cotrimoxazole, clindamycin, erythromycin, ciprofloxacin, gentamicin, vancomycin and linezolid and resistant to penicillin, tetracycline and cefoxitin (MRSA). M. Kaur et al,[18] Deepti et al,[15] Ramanathan Parameshwaran et al[16] and H. V. Patil et al[19] also showed that MRSA was responsible for majority cases of CRBSI.

\section{CONCLUSION}

CVCs are increasingly used in the inpatient and outpatient setting to provide longterm venous access. Duration of catheterisation and catheter colonisation has an important role in development of CRBSI which may lead to septicaemia. While inserting CVC preventive measures should be used like local antibiotics and catheter lock solution. Duration of catheterisation increases the morbidity, so regular monitoring of catheterised patient should be done for both insertion site and general condition.

This prospective study has highlighted the incidence, aerobic bacteriological profile of CVC infections and also the changing trends in the susceptibility pattern of the isolates to routinely used antibiotics in our tertiary care centre.

In our hospital, CRBSI rate is low (2.81 per 1000 catheter days) which is within normal range as per National Nosocomial Infection Surveillance System (NNIS). Proper training programme of hospital staff, maintenance of proper hand hygiene, following up of proper guidelines of catheter insertion and maintaining aseptic precaution prior to CVC insertion can reduce CRBSI.
Combined effort of the Microbiologists, clinicians and hospital infection control team are needed to tackle CRBSI.

\section{REFERENCES}

[1] Abad CL, Safdar N. Catheter-related Blood stream Infections. Infectious diseases. Special edn. New York, US: McMahon Publishing 2011:84-98.

[2] Edmond MB, Wallace SE, McClish DK, et al. Nosocomial bloodstream infection in United States hospitals: a 3 years analysis. Clinical Infectious Disease 1999;29(2):239-44.

[3] Pittet D, Tarara D, Wenzel RP, et al. Nosocomial bloodstream infection in critically ill patients. Excess length of stay, extra costs and attributable mortality. JAMA 1994;271(20):1598-601.

[4] Storti A, Manzato AJ, Pizzolitto AC, et al. Assessment of central venous catheter associated infections using semi quantitative or qualitative culture methods. Journal of Basic and Applied Pharmaceutical Sciences 2006;27(3):213-20.

[5] Maki DG, Weise CE, Sarafin HW. Semi quantitative culture method for identifying intravenous catheterrelated infection. New England Journal of Medicine 1977;296(23):1305-9.

[6] Frasca D, Dahyot-Fizelier C, Mimoz O. Prevention of central venous catheter-related infection in the intensive care unit. Critical Care 2010;14(2):212.

[7] Akan OA. Microorganisms isolated from catheter tip cultures: Ibni Sina hospital. Journal of Ankara Medical School 2003;25(3):113-118.

[8] Karchmer AW. Nosocomial bloodstream infections: organism, risk factors and implications. Clinical Infectious Disease 2000;31(Suppl 4):S139-S43.

[9] Pittet D, Wenzel RP. Nosocomial blood stream Infections: trends in rates, mortality and contribution to total hospital deaths. Arch Internal Medicine 1995;155(11):1177-84.

[10] Konemann EW, Allen SD, Janda WM, et al. Colour atlas and textbook of diagnostic microbiology. $4^{\text {th }}$ edn. Philadelphia: Lippincott Company 2011.

[11] Clinical and Laboratory Standards Institute. Performance standards for antimicrobial susceptibility testing. Twenty-Second informational supplement. 2012;32(3):M100-S22.

[12] Mermel LA, Allon M, Bouza E, et al. Clinical practice guidelines for the diagnosis and management of intravascular catheter-related infection: 2009 update by the infectious diseases society of America. Clinical Infectious Diseases 2009;49(1):1-45.

[13] National Nosocomial Infection Surveillance (NNIS) system report, data summary from January1992 to June 2002, issued August 2002. American Journal of Infection Control 2002;30(8):458-75.

[14] Singh S, Pandya Y, Patel R, et al. Surveillance of deviceassociated infections at a teaching hospital in rural Gujarat-India. Indian Journal of Medical Microbiology 2010;28(4):342-7.

[15] Deepti, Sinha S, Sharma SK, et al. Central venous catheter related blood stream infection in medical intensive care unit patients in a tertiary referral centre. Indian Journal of Chest Disease and Allied Science 2014;56(2):85-91. 
[16] Parameshwaran R, Sherchan JB, Varma DM, et al. Intravascular catheter related infection in an Indian tertiary care hospital. Journal of Infection in Developing Countries 2011;5(6):452-8.

[17] Bicudo D, Batista R, Furtado GH, et al. Risk factors for Catheter-related bloodstream infections: a prospective multi centres study in Brazilian intensive care units. Brazilian Journal of Infectious Disease 2011;15(4)32831.

[18] Kaur M, Gupta V, Gombar S, et al. Incidence, risk factors, microbiology of venous catheter associated blood stream infections - a prospective study from a tertiary care hospital. Indian Journal of Medical Microbiology 2015;33(2):248-54.
[19] Patil HV, Patil VC, Ramteerthkar MN, et al. Central venous catheter related blood stream infection in the intensive care unit. Indian Journal of Critical Care Medicine 2011;15(4):213-23.

[20] O'Connor A, Hanly AM, Francis E, et al. Catheter-related bloodstream infections in patients receiving parenteral nutrition: a prospective study of 850 patients. Journal of Clinical Medicine Research 2013;5(1):18-21.

[21] Varagali MA, Metgud SC, Kanadesai SG. Bacteriological profile of catheter tips and their antibiogram. National Journal of Basic Medical Sciences 2012;11(4):333-5. 